\title{
Serum proteome profiling of primary breast cancer indicates a specific biomarker profile
}

\author{
DANIEL BÖHM $^{1 *}$, KSENIA KELLER $^{1^{*}}$, NELLI WEHRWEIN ${ }^{2}$, ANTJE LEBRECHT $^{1}$, \\ MARCUS SCHMIDT ${ }^{1}$, HEINZ KÖLBL ${ }^{1}$ and FRANZ-HERMANN GRUS ${ }^{2}$ \\ Departments of ${ }^{1}$ Obstetrics and Gynecology, ${ }^{2}$ Experimental Ophthalmology, University Medical Center \\ of the Johannes Gutenberg University Mainz, Langenbeckstrasse 1, 55131 Mainz, Germany
}

Received May 28, 2011; Accepted July 1, 2011

DOI: 10.3892/or.2011.1420

\begin{abstract}
Non-invasive biomarkers for early breast cancer detection are urgently needed, as the risk of recurrent morbidity and mortality is closely related to the stage of the disease at the time of primary surgery. Currently, there are no established clinical biomarkers for breast cancer. Evaluation of protein expression patterns in body fluids using proteomic technologies can be used to discover new biomarkers for the detection of breast cancer. The aim of this study was to identify a biomarker signature identifying primary non-metastatic breast cancer and healthy controls. We screened 91 serum samples including 45 breast cancer patients and 46 healthy women using a proteomic approach. We found 14 biomarkers whose combination detects breast cancer patients from non-cancer controls with a sensitivity of $89 \%$ and specificity of $67 \%$. Five biomarkers were comparable with previously identified proteins from published data using similar approaches. This biomarker panel allows accurate discrimination between breast cancer and healthy individuals. In addition, it could distinguish subgroups of breast cancer based on patterns of several specific biomarkers. Further validation of biomarkers could potentially facilitate the early diagnosis of breast cancer as an aid to imaging diagnostics.
\end{abstract}

\section{Introduction}

The early detection of small breast cancers significantly improves survival rates; if breast cancer is diagnosed and treated while it is limited to the breast, the cure rate approaches $100 \%$ (1). Over $75 \%$ of women with breast cancer are older than 50

Correspondence to: Dr Daniel Böhm, Department of Obstetrics and Gynecology, University Medical Center of the Johannes Gutenberg University Mainz, Langenbeckstrasse 1, 55101 Mainz, Germany

E-mail: dboehm@uni-mainz.de

*Contributed equally

Key words: breast cancer, serum, proteome profiling, biomarker discovery, SELDI-TOF, MALDI-TOF-TOF years of age when they are diagnosed, and incidence rates have risen substantially over the last 10 years (2). Mortality rates from breast cancer have decreased due to the combination of earlier detection and improved adjuvant treatment regimens (3). Mammographic screening is currently the best available approach for early detection of breast cancer in women over the age of 50 years. Despite the fact that image resolution continues to improve through the use of digital technology, tumors $<5 \mathrm{~mm}$ are usually not detectable. Furthermore, up to $30 \%$ of mammographically detected tumors in women undergoing regular screening are disseminated to regional lymph nodes or distant metastasis at initial diagnosis. Mammography is also less sensitive in young women because of the increased density of breast tissue (4). High growth rate tumors might not be detected between the time intervals of screening mammography. Thus, new diagnostic modalities sensitive to early-stage breast cancer are needed to improve detection and increase the survival rate $(5,6)$.

The great opportunity for molecular tools to improve breast cancer outcomes based on early diagnosis has driven the search for new biomarkers. Previously identified blood-based breast cancer biomarkers include cancer antigen 15.3 (CA15.3) and carcinoembryonic antigen (CEA). Although these proteins are useful in combination with imaging and physical examination for monitoring ongoing treatment in breast cancer patients with metastatic disease, they lack sensitivity in detecting primary breast cancer at an early stage (7). The great heterogeneity of breast cancers suggests that more than one biomarker is needed for early detection (8). Therefore, the study of plasma or blood serum for patterns of expression of up to 10,000 different proteins is of great interest; this analysis places great demands on any methodology to be applied to plasma or serum proteome analysis. Many biomarkers of potential value for the diagnosis of breast cancer have not yet been detected, characterized, or evaluated due to their low concentration and subsequent difficulty in identification as they are covered by high abundant serum proteins such as albumin $(9,10)$.

A promising approach to biomarker discovery in proteomic technologies is the surface-enhanced laser desorption and ionization time-of-flight mass spectrometry (SELDI-TOF MS) or matrix-assisted laser desorption and ionization time-of-flighttime-of-flight mass spectrometry (MALDI-TOF-TOF MS). These analytical methods offer the ability to simultaneously 
profile hundreds of proteins in tissue, urine, or serum samples across a wide range of molecular weights $(11,12)$. Different comparative studies using this proteome-based approach have determined specific and significant protein patterns in breast cancer, but the use of new biomarkers for clinical applications is still under critical review. Many issues have to be addressed concerning the specificity of newly discovered biomarkers and the reproducibility of published data $(12,13)$.

We hypothesized that a panel of biomarkers of various specificities that were estimated after a combination of mass spectrometry techniques such as SELDI-TOF and MALDITOF-TOF should provide better sensitivity and specifity to breast cancer than a single assay. The aim of this monocentric clinical prospective study was to identify a biomarker signature in patients newly diagnosed with primary invasive breast cancer to assess its diagnostic potential in accurately identifying breast cancer patients (BC) and healthy individuals. The sensitivity and specificity of the results are reported. The molecular weights of identified proteins and their subunits were compared with previously discovered biomarkers.

\section{Materials and methods}

Study participants. This study enrolled 91 age-matched participants. Forty-five patients were diagnosed with primary breast carcinoma: one of these was diagnosed with ductal carcinoma in situ (DCIS). All patients were treated at the Department of Obstetrics and Gynecology, University Medical Center of the Johannes Gutenberg University Mainz. Forty-six healthy control females treated in the University Clinical Centre Mainz without any known malignancies were included. The breast cancer patients were routinely diagnosed by mammography and ultrasound guided core needle biopsy. Cancer patients were all treated with surgery and systemic therapy according to the national guidelines at the time. All healthy controls underwent mammography and ultrasound of the breast at the time of study inclusion. All samples were collected after the study members provided written informed consent for voluntary participation. The institutional ethics committee approved the study protocol in accordance with the ethical standard of the Declaration of Helsinki (1964).

Sample preparation. Venous blood was obtained from each patient prior to surgery with the use of plastic tubes (Serum- Monovetten ${ }^{\circledR}$, Sarstedt, Germany) with clot activator. Within the same time period, sera from healthy age-matched volunteers were collected. The preparation of serum samples was performed under strict, identical conditions for all study participants. The collected blood samples were allowed to clot for $15 \mathrm{~min}$ and then centrifuged for $5 \mathrm{~min}$ at $4^{\circ} \mathrm{C}$ and $3280 \mathrm{x} \mathrm{g}$. Sera were transferred into fresh plastic tubes and immediately frozen at $-80^{\circ} \mathrm{C}$ until further analysis.

SELDI-TOF and MALDI-TOF-TOF analysis. All experiments were accomplished within two weeks to reduce measurement fluctuations. Serum proteins were enriched using super paramagnetic beads with three different properties: magnetic bead-hydrophobic interaction chromatography (MB-HIC) C8 and $\mathrm{C} 18$ beads coated with hydrophobic residues and WCX beads acting as a weak cation-exchange tool (all purchased from Bruker Daltonics, Bremen, Germany). All elution steps were performed in accordance with annexed protocols provided by the manufacturer. Each sample $(2 \mu \mathrm{l})$ was analyzed on ProteinChip Array spots (Ciphergen Biosystems Inc.). The spots possessed different chromatographic surfaces: the reversed-phase (H50) and the weak cation exchange surface (CM10). All measurements were performed in a PBS-IIc ProteinChip Reader equipped with an AutoLoader after coating the eluates with sample matrix solution $(20 \mathrm{mg}$ sinapinic acid in $50 \%$ acetonitrile and $0.1 \%$ trifluoroacetic acid). Each sample was measured at low and high laser intensities to achieve the most optimal conditions for high accuracy measurement. For the low laser intensity (180), optimized for the best resolution of lower molecular weight proteins, the deflector setting of 1500 Da was used. For higher molecular weight proteins, the high laser intensity (210) and a deflector of 10,000 Da were applied. The mass-to-charge ratio $(\mathrm{m} / \mathrm{z})$ of the proteins generated in SELDI-TOF-MS was based on behalf of the external calibrants separately for both laser intensities.

The enriched serum proteins were also measured in a MALDI-TOF-TOF mass spectrometer (Ultraflex II; Bruker Daltonics, Bremen, Germany). Each sample (1 $\mu \mathrm{g})$ was put on $0.5 \mu \mathrm{l}$ of prespotted crystallization matrix (20 mg $\alpha$-cyano-4hydroxycinnamic acid in $50 \%$ acetonitrile and $2 \%$ trifluoroacetic acid) and coated after drying again with $0.5 \mu \mathrm{l}$ matrix. All samples were measured on two spots of MTP AnchorChip 600-384 target.

Data acquisition. Raw spectra from both mass spectrometers were transferred into Ciphergen Express ${ }^{\mathrm{TM}}$ Data Manager Software version 2.1 for the subsequent normalization and peak detection. The spectra derived from MALDI-TOF-TOF MS and SELDI-TOF MS were normalized separately. Using the detected peaks, clusters according to the different given conditions (e.g. laser intensity, the bead type used) were generated across all processed spectra. A peak cluster was created when the peak was found in $10 \%$ of all spectra for a given condition. After clustering, the data were exported together into Statistica (version 8.0; StatSoft, Tulsa, OK, USA) in the *csv file format. The estimated peak clusters consisted of the normalized peak intensities from every study participant.

Statistical analysis. The normalized intensities of the peaks with a certain molecular weight from all study members were compared across all conditions with the aid of t-tests and multivariate discrimination analysis testing the zero-hypothesis. The most significant biomarkers which distinguished BC and control (CTRL) groups were established for each condition. In the next step, the most precise protein biomarker signature from all given and tested conditions was determined from the combined biomarkers. For a comparison of protein peak intensity differences in cancer and CTRL participants, an analysis of variance (ANOVA) test was performed. To estimate the diagnostic potential of the final biomarker combination, an integrated artificial neural network (ANN) panel in Statistica software package was used. As input values, the peak intensities of the final biomarker which were significantly differentially regulated in BC and CTRL were chosen. The obtained output values demonstrated whether a cancer patient could be classified 
Table I. Characteristics of study participants.

\begin{tabular}{lc}
\hline & $\begin{array}{c}\text { Breast cancer patients } \\
\mathrm{n}=45\end{array}$ \\
& $59.4(39-84)$ \\
Mean age, years (range) ${ }^{\mathrm{a}}$ & \\
Tumor size, $\mathrm{n}(\%)$ & $1(2)$ \\
pTis & $23(51)$ \\
pT1 & $18(40)$ \\
pT2 & $3(7)$ \\
pT3-4 & \\
Nodal status, $\mathrm{n}(\%)$ & $26(58)$ \\
Negative & $18(40)$ \\
Positive & $7(16)$ \\
Tumor grade, $\mathrm{n}(\%)$ & $19(42)$ \\
Well differentiated & $19(42)$ \\
Moderately differentiated & \\
Poor/undifferentiated & \\
Estrogen receptor, $\mathrm{n}(\%)$ & $35(78)$ \\
Positive & $10(22)$ \\
Negative & \\
Progesterone receptor, $\mathrm{n}(\%)$ & $30(67)$ \\
Positive & \\
Negative & \\
HER2neu, $\mathrm{n}(\%)$ & \\
Positive & \\
Negative & \\
\hline & \\
& \\
&
\end{tabular}

${ }^{\text {a}}$ Healthy controls $(\mathrm{n}=46)$ : $58.7(36-85)$ years.

as such or not. For that purpose 150 artificial neuronal networks were used. The data set was split randomly and $50 \%$ of the data served as a train test. The second half of the data which was not provided into the train set was used for the test run. Finally, a receiver operating characteristic (ROC) curve was generated, plotting the sensitivity against the reciprocal specificity. The diagnostic accuracy of the determined biomarkers is reflected by the area under the ROC curve (AUC) which has a value of 1 in the case of a perfect classification.

\section{Results}

Table I reports the tumor characteristics of the breast cancer patients. Upon proteomic analysis, each sample showed a complex protein pattern, and a variety of proteins that differed between the clinical groups was detected. In order to find a biomarker panel which best differentiates between breast cancer and healthy patients, the SELDI-TOF and MALDITOF-TOF spectra were analyzed by multivariate statistical analysis. The ANOVA test (Fig. 1) revealed a protein biomarker profile with a statistical significant difference between both groups using the biomarker candidates from all conditions and surfaces simultaneously $(\mathrm{p}<0.05)$. The profile was defined by 14 peaks with a significant intensity difference: $9427 \mathrm{Da}$

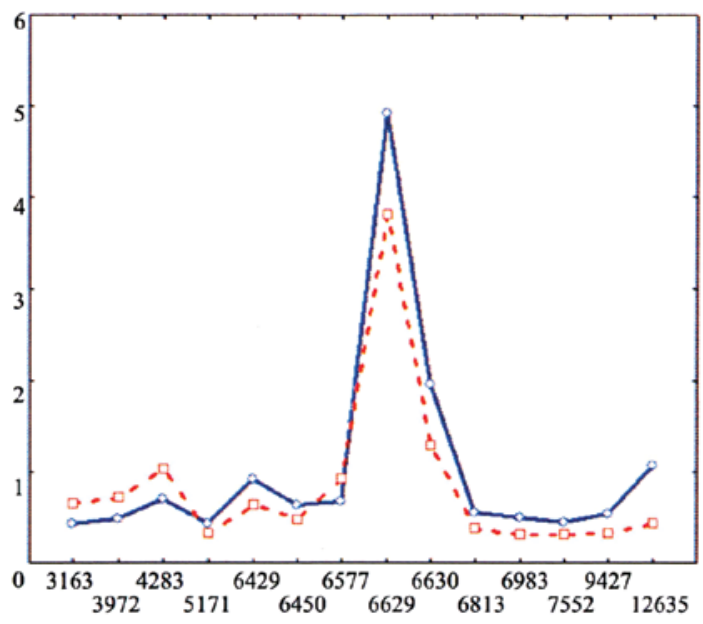

Figure 1. Biomarkers detected by SELDI-TOF MS. The X-axis represents the different molecular weights (in Da) of biomarkers which show the most significant differences between the groups (CTRL, blue line; BC, red line). The Y-axis shows the normalized intensity levels of the protein peaks.

$(\mathrm{p}=0.0035), 3163 \mathrm{Da}(\mathrm{p}=0.0117), 3972 \mathrm{Da}(\mathrm{p}=0.0131), 6630 \mathrm{Da}$ $(\mathrm{p}=0.0184), 6577 \mathrm{Da}(\mathrm{p}=0.0222), 6429 \mathrm{Da}(\mathrm{p}=0.0236), 6813 \mathrm{Da}$ $(\mathrm{p}=0.0259), 6983 \mathrm{Da}(\mathrm{p}=0.0289), 12,635 \mathrm{Da}(\mathrm{p}=0.0341)$, $4283 \mathrm{Da}(\mathrm{p}=0.0404), 7552 \mathrm{Da}(\mathrm{p}=0.0452), 6450 \mathrm{Da}(\mathrm{p}=0.0461)$, $6629 \mathrm{Da}(\mathrm{p}=0.0467)$ and $5171 \mathrm{Da}(\mathrm{p}=0.0480)$. Fig. 2 shows the categorized whisker plots from several of the peaks in the biomarker panel. Using this multimarker panel for input, an artificial neural network was trained with a training data set. The performance of the trained net was assessed using a test data set as described above (50\% split train:test). Based on the differentially regulated peaks in breast cancer and CTRL we were able to discriminate both groups with a high specificity $(67 \%)$ and sensitivity (89\%). Fig. 3 shows the ROC curve with an area under the curve (AUC) of 0.8 .

In addition, we aimed to identify biomarkers specific for subgroups of patients with various established breast cancer prognostic factors. Using the previously described ANOVA test we compared subgroups with different primary tumor sizes (T1 vs. T2) and expression of the estrogen and/or progesterone receptor (HR-positive versus HR-negative). Furthermore, we stratified the patients into a low- [tumor grade G1 or tumor grade G2 with a low level of urokinase-type plasminogen activator (uPA) and plasminogen activator inhibitor (PAI-1)] and a high-risk group (tumor grade G3 or tumor grade G2 with a high level of uPA and PAI-1). For each comparison we found a significant biomarker panel that significantly $(\mathrm{p}<0.05)$ differentiated between subgroups. The corresponding ROC curves are also shown (Fig. 4).

\section{Discussion}

In the present study, we used high-resolution MS profiling (SELDI-TOF and MALDI-TOF) of human serum to detect a biomarker signature that differentiates patients with breast cancer from healthy controls. Based on the differentially regulated peaks in CTRL and breast cancer patients, we were able to discriminate both groups with a high specificity $(67 \%)$ and sensitivity (89\%). Our investigation was designed 

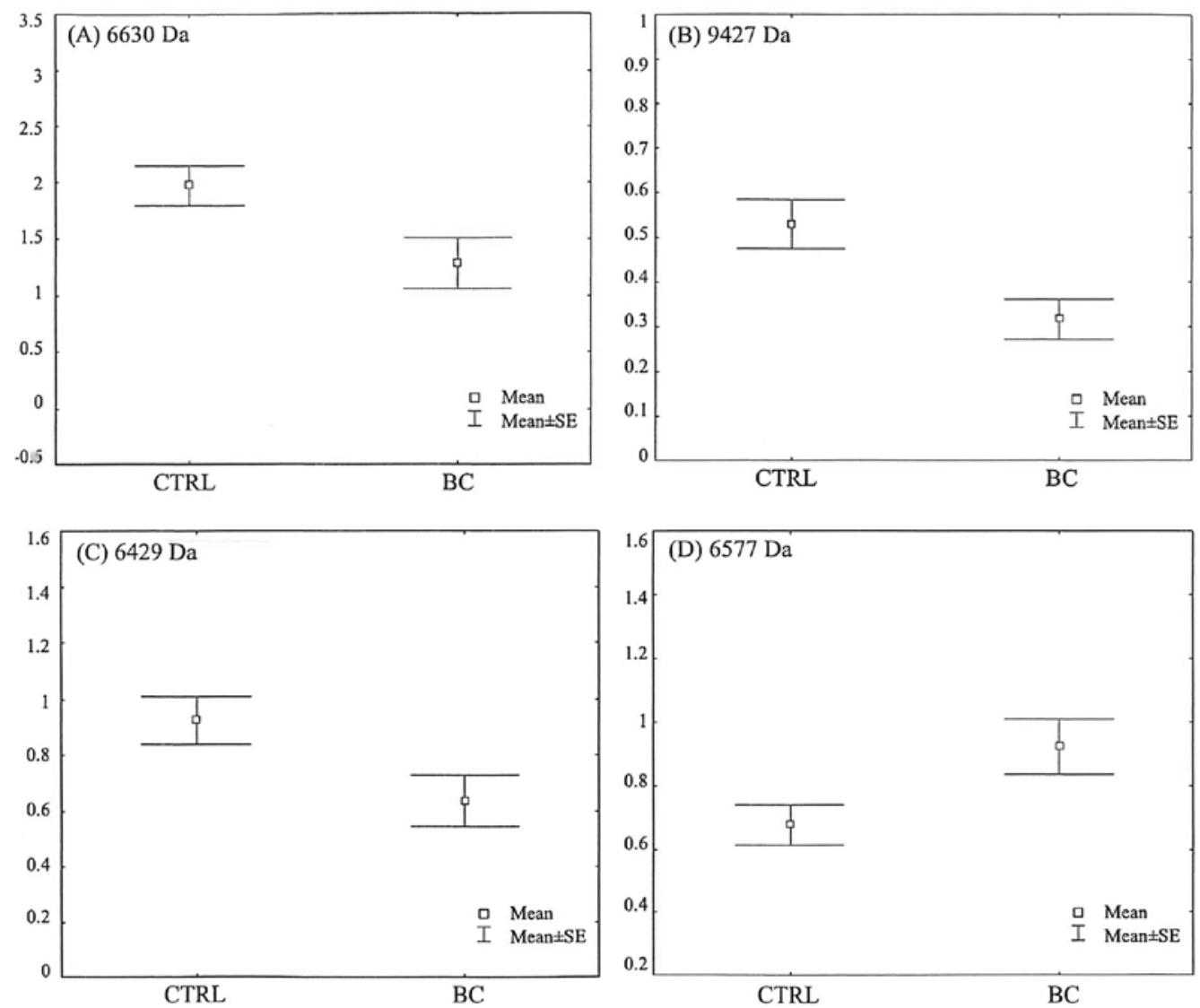

Figure 2. Categorized whisker plots. Several of the detected biomarkers are shown. In each plot, the $\mathrm{X}$-axis represents the two groups and the Y-axis represents the measured intensity values. Headers provide information concerning the molecular weight (in Da). (A) $6630 \mathrm{Da}(\mathrm{p}=0.0184)$; (B) 9427 Da ( $\mathrm{p}=0.0035$ ); (C) $6429 \mathrm{Da}(\mathrm{p}=0.0236)$, (D) $6577 \mathrm{Da}(\mathrm{p}=0.0222)$. CTRL, control group; BC, breast cancer patients.

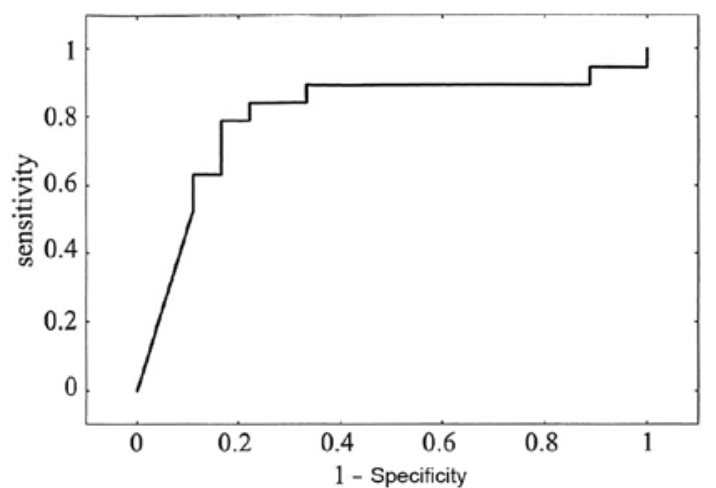

Figure 3. Based on the differentially regulated peaks in the control and breast cancer patients, we were able to discriminate both groups with a specificity of $63 \%$ and sensitivity of $90 \%$. An AUC of 0.8 was achieved. The X-axis represents sensitivity and Y-axis represents specificity.

to reduce bias to a minimum that may be caused by patient handling differences, sample collection, sample storage, and factors that were defined as important in the overall performance of an MS profiling based approach. This was achieved by restricting the collection and sample handling of all cases and controls to a single institution and by using the same personnel and procedures throughout the study. Furthermore, serum samples were not pretreated to deplete high-abundant proteins (e.g. albumin, immunoglobulin G).
Serum is the most readily available sample type for clinical proteomic studies. It may be a reliable surrogate tissue for analysis of physiologic or pathologic processes such as cancer. Some studies have addressed the potential of proteomic research to provide serum biomarkers that differentiate breast cancer from benign healthy controls (14-19). Our results compare well with previously reports by us and others, indicating high sensitivity and specificity when an MS profiling method is used for detecting breast cancer in readily accessible body fluids (20).

The present study focused on the detection of nonmetastatic lesions. Some of the biomarkers we found do not seem to overlap with findings in other published studies. The fact that we did not find comparable peptides is not surprising since differences in the used ProteinChip surface chemistry, binding/washing conditions, and instrument setting may all contribute to the binding and detection of different protein species and therefore lead to the discovery of different subsets of biomarkers. Removal of high-abundant proteins might lead to loss of biomarker candidates; for example albumin serves as a carrier for low molecular weight proteins. These variations could explain the differences in the novel published biomarkers for breast cancer by protein profiling. Although diversity in study protocols would facilitate the detection of a broader spectrum of proteins and therefore generate more candidate biomarkers, the strength of aforementioned peak entities remains weak due to the lack of validation (12). 

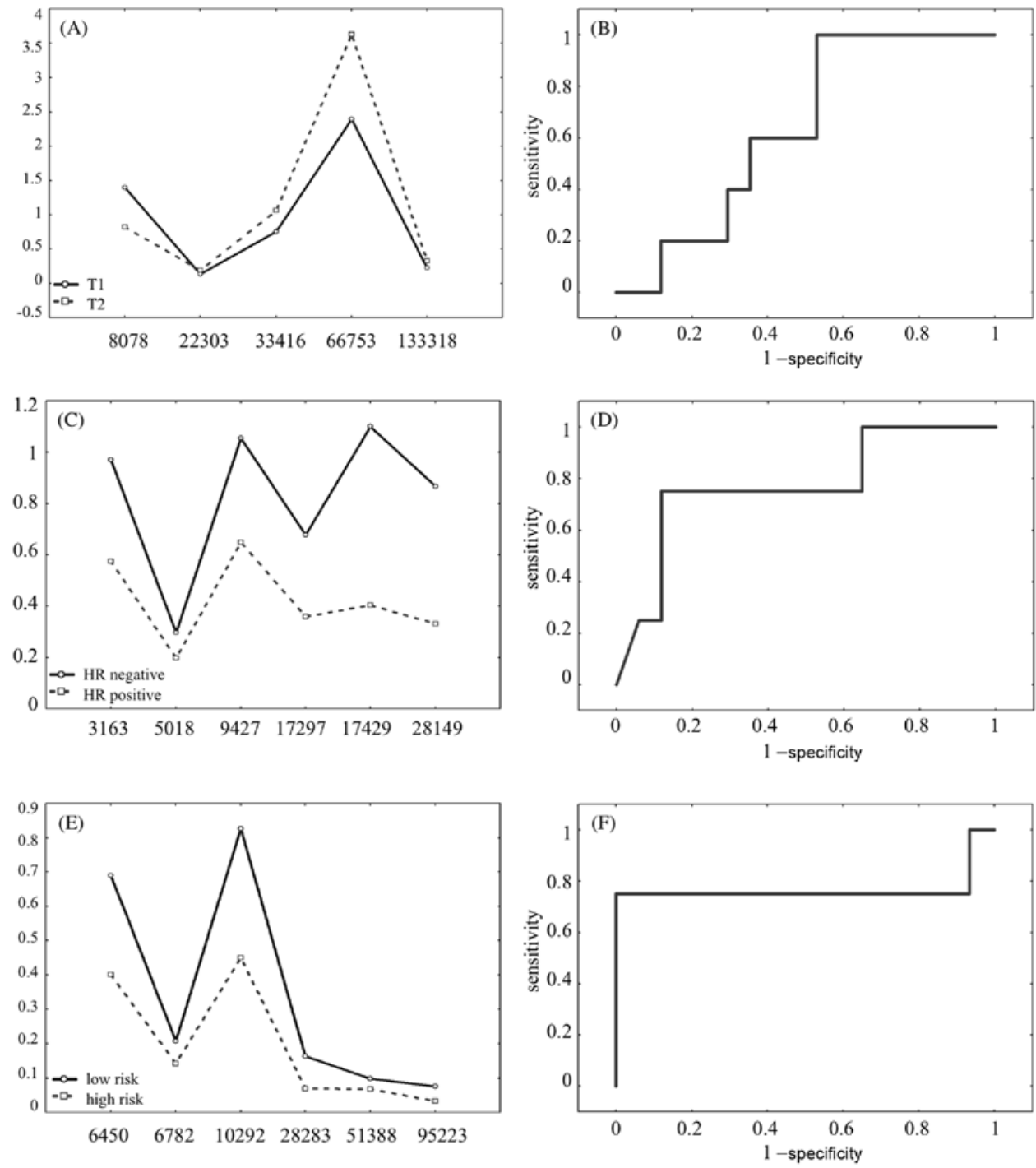

Figure 4. Based on the differentially regulated peaks in the defined subgroups, the biomarker profile and the corresponding ROC curves of each subgroup are shown. (A) Biomarker profile of subgroups T1 vs. T2. All biomarkers were detected with SELDI-TOF measurement. (B) ROC curve of T1 vs. T2 with sensitivity of $60 \%$ and specificity of 65\% (AUC: 0.64). (B) Biomarker profile of the HR-negative vs. HR-positive subgroup: 3163, 5018, and 9427 Da were detected with MALDI-TOF-TOF MS, others with SELDI-TOF MS. (D) ROC curve of the HR-negative vs. HR-positive subgroup with sensitivity of 75\% and specificity of $88 \%$ (AUC: 0.77). (E) Biomarker profile of the low-risk vs. high-risk subgroup; SELDI-TOF: 10,292, 28,283, 51,388 and 95,223 Da; MALDI-TOF-TOF: 6782 and $6450 \mathrm{Da}$. (F) ROC curve of the low-risk vs. high-risk subgroup with sensitivity of 75\% and specificity of 100\% (AUC: 0.77 ).

Furthermore, the heterogeneity of malignant cells and the variability of the host background create molecularly distinct subgroups of tumors having different phenotypes, resulting in numerous biomarkers for the early detection of cancer (21-23).

The potential use of a detection technology designed to identify breast cancer is evident: detection at an early stage remains an effective way to decrease breast cancer related mortality. Although mammography has improved early breast cancer diagnosis, it is far from optimal. The sensitivity of mammography ranges between 63 and 87\%, depending on age, breast density, and tumor characteristics (24). In particular, serum tumor markers of breast cancer (CA15.3, CEA) do not have enough sensitivity and specificity to apply them for diagnosis (7). Therefore, we aimed to identify serum proteome patterns specific for subgroups of patients with different clinical and molecular outcomes that may be helpful for selecting cases for more sensitive diagnostic techniques. Subgroups related to primary tumor size and expression of estrogen and/or proges- terone receptor were significantly differentiated. Of note, we were able to discriminate low-risk (tumor grade G1 or tumor grade G2 with a low level of UPA and PAI-1) and high-risk breast cancer patients (tumor grade G3 or tumor grade G2 with a high level of uPA and PAI-1) with a high sensitivity (75\%) and specificity (100\%). These findings may be used for increasing the positive predictive value of mammographically suspicious lesions or providing further information for patients who might receive neoadjuvant systemic therapy.

Serum protein profiling studies by MALDI-TOF-TOF or SELDI-TOF MS have yielded numerous protein peaks that provide accurate class discrimination between patients with breast cancer and healthy controls. However, only a small percentage of reported peaks have been structurally identified, although protein identification is essential for insights into molecular mechanisms.

By searching previously published data using similar approaches, we retrieved five protein peaks by comparing 
the obtained molecular masses with the molecular weights of identified proteins and their subunits. The biomarker candidate at 4283 and 3972 Da was described and identified as a fragment of inter- $\alpha$-trypsin inhibitor heavy chain 4 (ITIH4) by Song et al (25) in breast cancer patients and Peng et al (26) in prostate cancer patients. The peak at 3972 Da was compared to the identified ITIH4 by Villanueva et al and found to be increased in the sera of prostate and bladder cancer patients (27). Protein peaks at 6630 and 6629 Da were identified as apolipoprotein $\mathrm{C}-\mathrm{I}$ in the sera of patients with papillary thyroid carcinoma by Fan et al (28) and sera of colorectal cancer patients by Ward et al (29). The peak at 6429 Da has not yet been identified but was described as a putative biomarker in lung cancer by Yang et al (30). As these studies generally yielded contradictory results, further research is needed to determine the true value of these markers in breast cancer management.

In conclusion, our results demonstrated potential serum biomarker patterns in the low molecular weight region of the serum proteome that can properly segregate primary breast cancer patients from healthy controls. We support the promising techniques of SELDI-TOF and MALDI-TOF-TOF for biomarker discovery. Using the protein panel of 14 selected biomarkers, we achieved high sensitivity and specificity for the detection of invasive breast cancer. Further validation of the discovered biomarkers in an independent population can help to improve our understanding of cancer emergence and development and may facilitate the early diagnosis of breast cancer.

\section{Acknowledgements}

We acknowledge Nina Matheis for scientific support. This study was partially supported by funding from the Mainz Research Program (MAIFOR NR. 122), University Medical Centre Mainz.

\section{References}

1. Jensen AR, Madsen AH and Overgaard J: Trends in breast cancer during three decades in Denmark: stage at diagnosis, surgical management and survival. Acta Oncol 47: 537-544, 2008.

2. Muller-Schimpfle MP,Heindel W, Kettritz U,Schulz-Wendtland R and Bick U: [Consensus Meeting of Course Directors in Breast Imaging, 9 May 2009, in Frankfurt am Main - Topic: Masses]. Rofo 182: 671-675, 2010 (In German).

3. Schmidt M, Victor A, Bratzel D, et al: Long-term outcome prediction by clinicopathological risk classification algorithms in node-negative breast cancer - comparison between Adjuvant!, St Gallen, and a novel risk algorithm used in the prospective randomized Node-Negative-Breast Cancer-3 (NNBC-3) trial. Ann Oncol 20: 258-264, 2009.

4. Rosenberg RD, Lando JF, Hunt WC, et al: The New Mexico Mammography Project. Screening mammography performance in Albuquerque, New Mexico, 1991 to 1993. Cancer 78: 1731-1739, 1996.

5. Ugnat AM, Xie L, Morriss J, Semenciw R and Mao Y: Survival of women with breast cancer in Ottawa, Canada: variation with age, stage, histology, grade and treatment. Br J Cancer 90: 1138-1143, 2004.

6. Sant M, Allemani C, Capocaccia R, et al: Stage at diagnosis is a key explanation of differences in breast cancer survival across Europe. Int J Cancer 106: 416-422, 2003.

7. Harris L, Fritsche H, Mennel R, et al: American Society of Clinical Oncology 2007 update of recommendations for the use of tumor markers in breast cancer. J Clin Oncol 25: 5287-5312, 2007.
8. Desmetz C, Bascoul-Mollevi C, Rochaix P, et al: Identification of a new panel of serum autoantibodies associated with the presence of in situ carcinoma of the breast in younger women. Clin Cancer Res 15: 4733-4741, 2009.

9. Liotta LA, Ferrari M and Petricoin E: Clinical proteomics: written in blood. Nature 425: 905, 2003.

10. Liotta LA and Petricoin EF: Serum peptidome for cancer detection: spinning biologic trash into diagnostic gold. J Clin Invest 116: 26-30, 2006.

11. Ebert MP, Korc M, Malfertheiner P and Rocken C: Advances, challenges, and limitations in serum-proteome-based cancer diagnosis. J Proteome Res 5: 19-25, 2006.

12. Kiehntopf M, Siegmund R and Deufel T: Use of SELDI-TOF mass spectrometry for identification of new biomarkers: potential and limitations. Clin Chem Lab Med 45: 1435-1449, 2007.

13. Li J, Zhao J, Yu X, et al: Identification of biomarkers for breast cancer in nipple aspiration and ductal lavage fluid. Clin Cancer Res 11: 8312-8320, 2005

14. Pawlik TM and Kuerer HM: The evolving role of proteomics in the early detection of breast cancer. Int J Fertil Womens Med 50: 212-216, 2005.

15. Mathelin C, Cromer A, Wendling C, Tomasetto C and Rio M-C: Serum biomarkers for detection of breast cancers: a prospective study. Breast Cancer Res Treat 96: 83-90, 2006.

16. Li J, Zhang Z, Rosenzweig J, Wang YY and Chan DW: Proteomics and bioinformatics approaches for identification of serum biomarkers to detect breast cancer. Clin Chem 48: 1296-1304, 2002.

17. Belluco C, Petricoin EF, Mammano E, et al: Serum proteomic analysis identifies a highly sensitive and specific discriminatory pattern in stage 1 breast cancer. Ann Surg Oncol 14: 2470-2476, 2007.

18. van Winden AWJ, van den Broek I, Gast M-CW, et al: Serum degradome markers for the detection of breast cancer. J Proteome Res 9: 3781-3788, 2010.

19. Pietrowska M, Marczak L, Polanska J, et al: Mass spectrometrybased serum proteome pattern analysis in molecular diagnostics of early stage breast cancer. J Transl Med 7: 60, 2009.

20. Zhang GQ, Du J and Pang D: [Detection and clinical significance of serum proteomic patterns of breast cancers by surface enhanced laser desorption/ionization time of flight mass spectrometry]. Zhonghua Zhong Liu Za Zhi 28: 204-207, 2006 (In Chinese).

21. van de Vijver M, He Y, van't Veer L, et al: A gene-expression signature as a predictor of survival in breast cancer. $\mathrm{N}$ Engl $\mathrm{J}$ Med 347: 1999-2009, 2002.

22. Schmidt M, Hasenclever D, Schaeffer M, et al: Prognostic effect of epithelial cell adhesion molecule overexpression in untreated node-negative breast cancer. Clin Cancer Res 14: 5849-5855, 2008.

23. Ginestier C, Charafe-Jauffret E, Bertucci F, et al: Distinct and complementary information provided by use of tissue and DNA microarrays in the study of breast tumor markers. Am J Pathol 161: 1223-1233, 2002.

24. Elmore JG, Armstrong K, Lehman CD and Fletcher SW: Screening for breast cancer. JAMA 293: 1245-1256, 2005.

25. Song J, Patel M, Rosenzweig CN, et al: Quantification of fragments of human serum inter-alpha-trypsin inhibitor heavy chain 4 by a surface-enhanced laser desorption/ionization-based immunoassay. Clin Chem 52: 1045-1053, 2006.

26. Peng J, Stanley AJ, Cairns D, Selby PJ and Banks RE: Using the protein chip interface with quadrupole time-of-flight mass spectrometry to directly identify peaks in SELDI profiles - initial evaluation using low molecular weight serum peaks. Proteomics 9: 492-498, 2009.

27. Villanueva J, Shaffer DR, Philip J, et al: Differential exoprotease activities confer tumor-specific serum peptidome patterns. J Clin Invest 116: 271-284, 2006.

28. Fan Y, Shi L, Liu Q, et al: Discovery and identification of potential biomarkers of papillary thyroid carcinoma. Mol Cancer 8: 79, 2009 .

29. Ward DG, Suggett N, Cheng Y, et al: Identification of serum biomarkers for colon cancer by proteomic analysis. Br J Cancer 94: 1898-1905, 2006.

30. Yang SY, Xiao XY, Zhang WG, et al: Application of serum SELDI proteomic patterns in diagnosis of lung cancer. BMC Cancer 5: 83, 2005. 